\title{
ACTION DE DIFFÉRENTES DOSES DE CHLORURE ET SULFATE DE SODIUM SUR LA PRODUCTION DE LA POULE PONDEUSE ET LA QUALITÉ DE L'EUF (1)
}

\author{
M. AMIN $\left({ }^{2}\right)$, P. MONGIN et B. SAUVEUR \\ avec la collaboration technique de D. Dubors \\ Station de Recherches avicoles, \\ Centre de Recherches de Tours, \\ 37 - Nouzilly \\ Institut national de la Recherche agronomique
}

\section{RÉSUMÉ}

Le but de notre étude est de savoir dans quelle mesure les teneurs en chlore et en sulfate du régime affectent la production de la poulc pondeuse et la qualité de l'ouf lorsque l'apport de sodium reste constant. Nous avons effectué ces essais sur des poules Rhode Island Red $\times$ Wyandotte et enregistré le poids des animaux, l'intensité de ponte ainsi que le poids, la solidité de coquille et la qualité de l'albumen de l'œuf.

Une carence totale en chlore (avec apport sodé normal) entraîne en 5 semaines une perte de poids corporel de $4^{\circ 0} \mathrm{~g}$ ct une cessation à peu près complète de la ponte dès la $4^{\mathrm{e}}$ semaine (fig. I).

Avec une teneur en chlore du régime équivalente à $0, \mathbf{I}$ p. Ioo de $\mathrm{NaCl}$ (fig. 2 et 3) nous observons une baisse relative de l'intensité de ponte et du poids de l'œuf par rapport au lot témoin (- Io,8 p. Ioo et $-3,7 \mathrm{~g}$ respectivement après 7 semaines); de même, la qualité de l'albumen diminue (- 4,2 unités Haugh). A l'opposé, après deux semaines, le traitenent semble favorable à la solidité de la coquille mais la fréquence des taches de sang présentes dans l'œuf est multipliée par 7 .

L'apport de quantités variables de chlore et de sulfate effectué après la déficience chlorée montre que le besoin en chlore de la poule pondeuse est voisin de o, 2 p. Ioo de $\mathrm{NaCl}$ et qu'il existe une interaction entre ces deux anions. Lorsque l'apport de chlore atteint la norme ci-dessus, une supplémentation en sulfate de sodium de o,25 p. Ioo permet d'améliorer la qualité de la coquille sans nuire à celle de l'albumen ni aux autres paramètres étudiés. S'il subsiste en revanche une déficience en chlore, l'apport de sulfate en accentue les effets néfastes sur la qualité interne do l'œuf (unités Haugh et taches de sang).

La déficience en chlore provoquant en outre l'apparition d'une alcalose métabolique, les résultats ci-dessus sont discutés en rapport avec l'action de l'équilibre acido-basique sur la formation de la coquille et de l'albumen de l'ouf.

(1) Ce travail représente une partie de la thèse de Doctorat d'Université de M. Amin, soutenue à la Faculté des Sciences de Paris en juin 1968.

(2) Adresse actuelle : Faculté agronomique. Département de Zootechnie. Université de TéhéranKaradj (Iran).

Les tirés à part doivent être commandés à : B. Sauveur, Station de Recherches avicoles. Centre de Recherches de Tours, 37 - Nouzilly (France). 


\section{IN'TRODUC'TION}

I,e chlorure de sodium est la forme la plus économique d'apport du sodium nécessaire à toute production et on néglige généralement de savoir quel rôle propre peut jouer le chlore.

C'est ainsi que la bibliographie manque de données sur l'action des seuls ions chlorure du régime chez la poule pondeuse. On sait qu'une supplémentation en chlore diminue l'épaisseur et la solidité des coquilles (LöRCHER et al., I964) mais on ignore tout des besoins réels de la poule et des effets d'une restriction chlorée sur la qualité de la coquille ou celle de l'albumen de l'œuf lorsque l'apport de sodium reste normal.

Chez l'Homme, une déficience chlorée entraîne l'apparition d'une alcalose avec acidurie (RICHE'T et LISSAC, I963) et l'on sait qu'une telle situation peut être favorable à la qualité de la coquille de l'œuf (MongIN, I968 a) mais défavorable pour l'albumen (SAUVEUR, I970).

Par ailleurs, il a été montré que les ions sulfate peuvent participer indirectement au contrôle de la formation de l'œuf (SAUVEUR, r970) mais le niveau optimum de leur incorporation éventuelle dans un régime pour pondeuses n'a jamais été défini.

Le but de la présente étude est donc de savoir dans quelle mesure des taux variables d'ions chlorure et sulfate agissent sur la production et la qualité des œufs lorsque l'apport sodé reste constant. Pour aborder ce sujet, nous avons entrepris une étude préliminaire de la teneur en chlore des matières premières couramment

TABLEAU I $a$

Teneur en sodium, potassium et chlore des principales matières premières utilisées dans l'alimentation des volailles

(en g par kg)

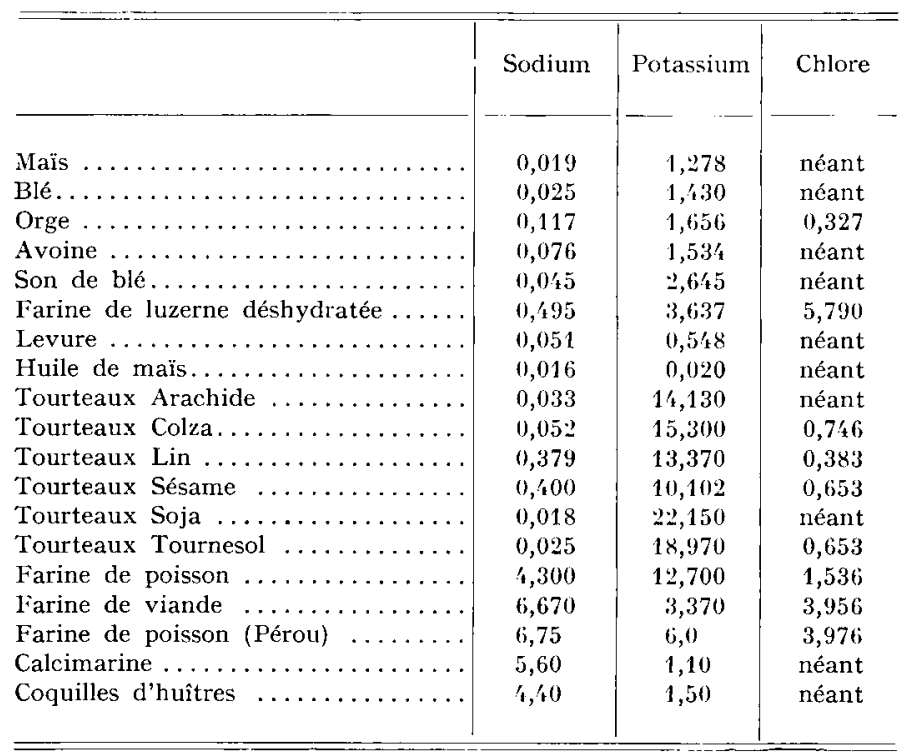


utilisées dans l'alimentation des volailles ; les résultats des analyses de chlore, sodium et potassium sont rapportées au tableau I $a$.

Puis, au moyen de régimes synthétiques, assurant un apport sodé normal, nous avons étudié successivement l'action d'une carence totale en chlore et d'une simple déficience. Ėnfin dans une troisième expérience, nous avons observé des effets de la restauration chlorée, complétée par l'apport de différentes doses de sulfate de sodium.

Nous rapportons ici les effets enregistrés sur divers paramètres zootechniques (poids des poules, taux de ponte, qualité de la coquille et de l'albumen de l'œuf) ; l'analyse des paramètres sanguins et de la composition électrolytique de l'albumen est décrite par ailleurs.

\section{MATÉRIEL ET MÉTHODES}

\section{Animaux et régimes}

\section{a) Expérience I. Carence totale en chlore.}

Cette expérience est réalisée sur 20 poules Rhode Island Red $\times$ Wyandotte âgées de 9 mois, placées en cages individuelles et entraînées à consommer l'aliment synthétique supplémenté en chlore en le mélangeant progressivement à l'aliment habituel ; la transition se fait ainsi sans heurt en 5 semaines. La composition du régime de base et du complément minéral est donnée aux tableaux I $b$ et I $c$. Cet aliment contient I $6 \mathrm{p}$. roo de protéines et 2800 calories métabolisables par $\mathrm{kg}$; l'analyse ne révèle aucune trace de chlorure dans le régime de base. Après la période d'adaptation, les animaux sont répartis en deux lots; l'un reçoit pendant 5 semaines (du 24 avril au 28 mai) le régime ci-dessus (lot carencé) et l'autre le même aliment supplémenté avec $380 \mathrm{~g}$ de $\mathrm{CaCl}_{2} /$ I oo $\mathrm{kg}$ soit l'équivalent en chlore de $0,4 \mathrm{p}$. Ioo de $\mathrm{NaCl}$ (norme $\mathrm{N}$. R. C.). A la suite de ces 6 semaines, les 2 lots reçoivent le régime normalement pourvu en chlore.

TABLFAU I $b$

Composition du végime (p. Ioo)

\begin{tabular}{|c|c|}
\hline Amidon & 56,4 \\
\hline Cérélose... & 6,0 \\
\hline 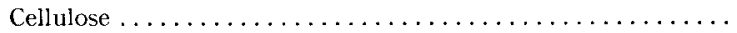 & 4,0 \\
\hline 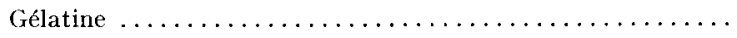 & 3,0 \\
\hline Drackett protein Soja $\ldots \ldots \ldots \ldots \ldots \ldots \ldots \ldots \ldots$ & 18,0 \\
\hline 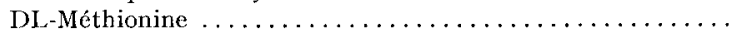 & 0,6 \\
\hline 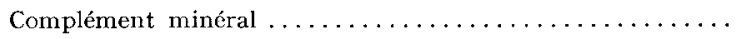 & $11,305(1)$ \\
\hline Complément vitaminique ...... & 0,699 \\
\hline
\end{tabular}

(1) Voir tableau $1 c$.

\section{b) Expérience II. Déficience chlorée.}

Pour la deuxième expérience, nous utilisons I 28 autres poules de même race. Après un mois d'adaptation au régime synthétique normochloré, les animaux sont répartis en 8 lots de I6; 1'un (lot T) ne subit aucune modification (teneur en chlore du régime équivalente à o, $4 \mathrm{p}$. 1oo de $\mathrm{NaCl}$ ) et les 7 autres ne reçoivent que l'équivalent de o, I p. Ioo de $\mathrm{NaCl}$ (sous forme de $\mathrm{CaCl}_{2}$ ). $\mathrm{La} \mathrm{com-}$ position du régime de base est la même que précédemment à l'exception des protéines isolées de soja, remplacées par du tourteau de soja, lui aussi totalement dépourvu de chlore. L'expérience 
TABLEAU I $c$

Complément minéral (pour Ioo $\mathrm{kg}$ d'aliment)

\begin{tabular}{|c|c|c|}
\hline$\ldots \ldots \ldots \ldots \ldots \ldots$ & 8,0 & $0 \mathrm{~kg}$ \\
\hline 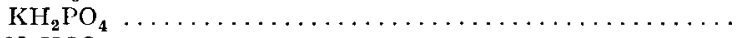 & 2,4 & $\mathrm{~kg}$ \\
\hline 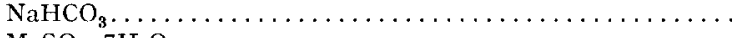 & 0,4 & $\operatorname{kg}$ \\
\hline 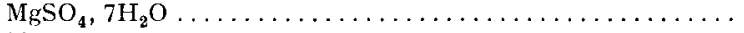 & 250 & g \\
\hline 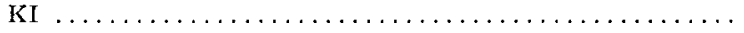 & 4 & $\mathrm{~g}$ \\
\hline 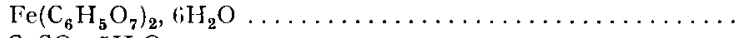 & $1 / 0$ & $g$ \\
\hline 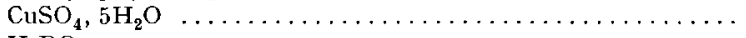 & 2 & $g$ \\
\hline 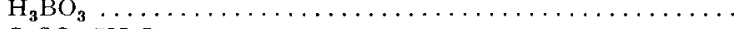 & 0,9 & $g$ \\
\hline 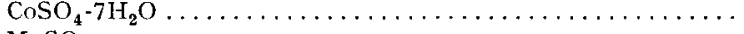 & 0,1 & $g$ \\
\hline$\ldots \ldots \ldots \ldots \ldots \ldots \ldots \ldots \ldots \ldots \ldots \ldots$ & 65 & g \\
\hline$\ldots \ldots \ldots \ldots \ldots \ldots \ldots \ldots \ldots \ldots \ldots \ldots \ldots \ldots$ & 44 & $\mathrm{~g}$ \\
\hline
\end{tabular}

dure alors 7 semaines (du 23 novembre au 12 janvier). Dans les deux expériences, un apport normal de sodium est assuré à tous les lots (témoins et carencés) par une supplémentation en $\mathrm{NaHCO}_{3}$ (cf. tabl. I c).

\section{c) Expérience III. Apport de chlorure et de sulfate de sodium.}

Cette expérience est la suite chronologique de la précédente et porte donc sur les mêmes animaux divisés en 2 groupes de 4 lots comme l'indique le tableau I $d$. Le régime de base est le même que précédemment dans lequel nous introduisons un mélange de chlorure et de sulfate de sodium en proportions variables de telle façon que l'apport de sodium soit constamment de

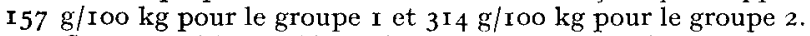

Cette expérience s'étend à nouveau sur une période de 7 semaines ( 13 janvier-3 mars).

\section{TABLEAU I $d$}

Apport de $\mathrm{NaCl}$ et $\mathrm{Na}_{2} \mathrm{SO}_{4}$ à chacun des 8 lots lors de l'expérience III (g/roo kg d'aliment)

\begin{tabular}{|c|c|c|c|}
\hline Lots & $\mathrm{NaCl}$ & $\mathrm{Na}_{2} \mathrm{SO}_{4}$ & $\mathrm{Na}$ \\
\hline \multicolumn{4}{|l|}{ Groupe 1} \\
\hline 1 & 100 & 30.3 & 1.57 \\
\hline 2 & 200 & 212 & 157 \\
\hline 3 & 300 & 121 & 157 \\
\hline '́t & 400 & 0) & 157 \\
\hline \multicolumn{4}{|l|}{ Groupe 2} \\
\hline 5 & 100 & 817 & $31^{\prime}$ \\
\hline 6 & 200 & $7: 96$ & $31^{\prime}$ \\
\hline 7 & 300 & 605 & $31^{\prime}$ \\
\hline 8 & 400 & 隹 & $31^{\prime} \mathrm{t}$ \\
\hline
\end{tabular}

\section{Analyses}

Dans chacune des 3 expériences nous cnregistrons le poids des oufs et des poules ainsi que leur intensité de ponte $=$ (nombre d'œufs pondus par jour/nombre de poules présentes) $\times$ Ioo. Lors des expériences II et III, la solidité de la coquille est appréciée indirectement en mesurant le poids de coquille par Ioo $\mathrm{cm}^{2}$ de surface ("index de coquille " de MongIN, I965) ; la hauteur de l'albumen ferme est exprimée en unités de HaUGH (1937) et la fréquence des oufs présentant des taches de sang en p. Ioo de la production totale. 


\section{Analyses statistiques}

L'analyse statistique est conduite pour chaque essai au moyen d'une analyse de variance globale des résultats et à l'aide de tests de $t$ de Student. Comme très souvent la variation interpoules à l'intérieur d'un même lot est supérieure à la variation due au traitement, chaque animal est comparé à lui-même et nous transformons les résultats bruts en exprimant les différences observées entre chaque valeur enregistrée au cours de la période expérimentale et la moyenne des valeurs relevées lors du pré-traitement ou à la fin du traitement précédent (modèle utilisé pour toutes les caractéristiques de l'cuf).

\section{RÉSULTATS}

\section{Expérience I. Carence totale en chlore}

(o vs. 0,4 p. Ioo de $\mathrm{NaCl}$ )

Les résultats essentiels sont regroupés sur la figure I. Nous voyons (fig. I-I) qu'une carence totale en chlore fait diminuer rapidement le poids vif des animaux;
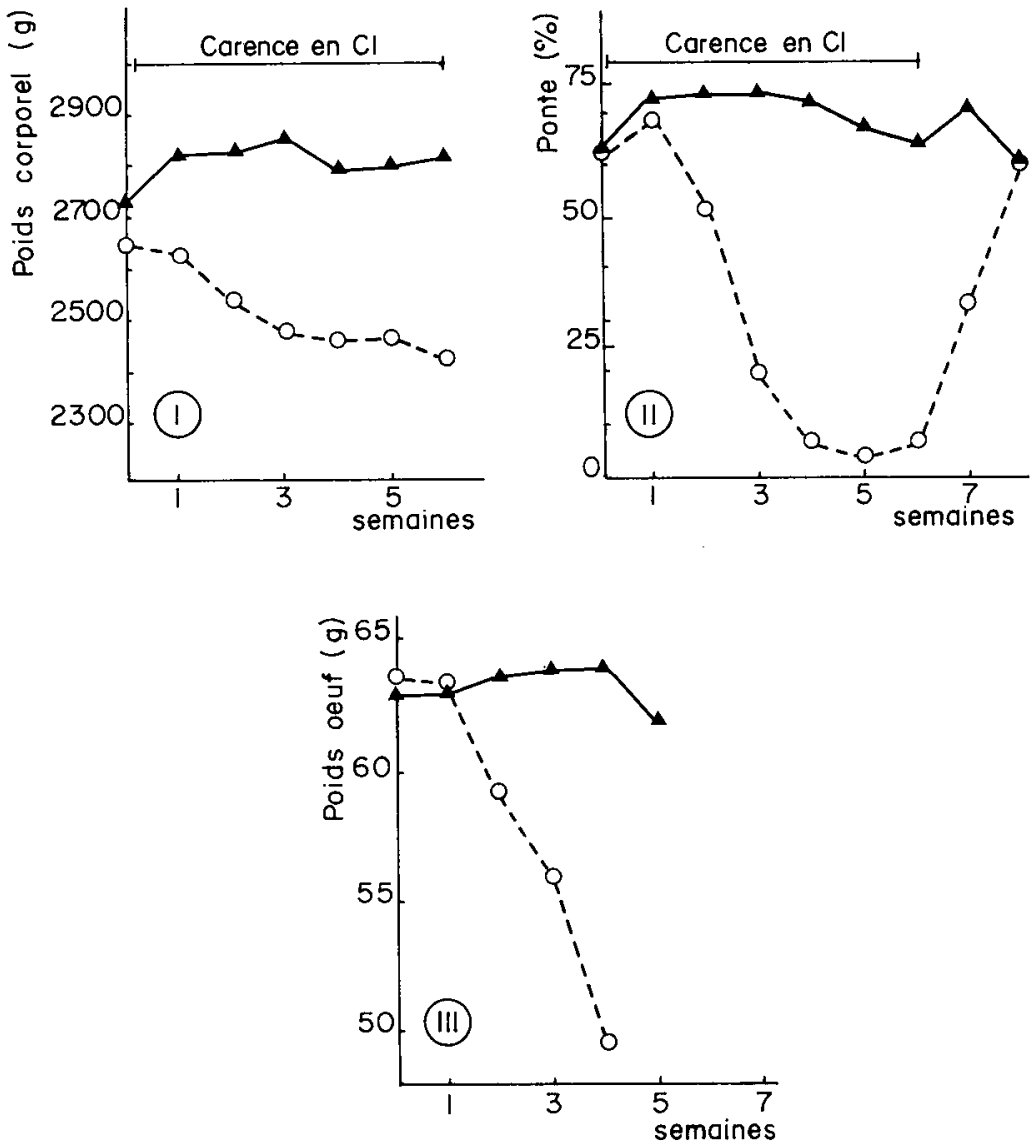

FIG. I. - Carence totale en chlore. Action sur le poids corporel des poules (I). l'intensité de ponte (II) et le poids de l'ouf (III)

^__ lot Témoin $(0,24 \mathrm{p}$. Ioo $\mathrm{Cl})$

..... o lot carencé en $\mathrm{Cl}$ (o p. roo $\mathrm{Cl}$ ) 
dès la deuxième semaine, la chute est en moyenne de 250 g. Elle s'accentue ensuite puis le poids semble se stabiliser à la $4^{\mathrm{e}}$ semaine de carence. Après 5 semaines de ce régime, la perte de poids approche $400 \mathrm{~g}$ par poule $(\mathrm{P}<0,0005)$.

L'intensité de ponte (fig. I-II) accuse une très nette diminution sous l'action de la carence chlorée et se stabilise aux environs de $5 \mathrm{p}$. Ioo dès la $4^{\mathrm{e}}$ semaine. Cette baisse considérable de production est due pour partie à l'arrêt total de la ponte de certains animaux mais tous sont affectés par la carence.

Comme la production, le poids de l'œuf s'effondre (fig. I-III) dès la deuxième semaine de carence. Bien que le nombre d'œufs soit très faible à partir de la $3^{\mathrm{e}}$ semaine, les différences entre lots témoins et traités sont significatives.

La restitution du chlore alimentaire restaure la ponte en deux semaines (fig. I-II) ce qui montre l'effet propre de cet élément. Cette expérience préliminaire nous permet donc d'affirmer, pour la suite de notre travail, que les variations des paramètres étudiés seront dues à la déficience en ions $\mathrm{Cl}^{-}$.

\section{Expérience II. Déficience en chlore (o,I vs. 0.4 p. Ioo de $\mathrm{NaCl}$ )}

Les résultats obtenus au cours des 7 semaines de déficience chlorée sont représentés par les figures 2 et 3 , donnant les valeurs enregistrées pour les divers paramètres à plusieurs stades du traitement ( $\mathrm{I}, 2,3,6$ et 7 semaines).

Le tableau 2 indique, pour chaque stade, les différences entre l'ensemble des lots restreints et le lot témoin et leur seuil de signification ; il donne également les résultats de l'analyse de variance globale lorsque celle-ci a été effectuée ; enfin, nous y avons reporté les valeurs de la dernière semaine exprimées en p. Ioo de celles enregistrées lors de la première observation afin de mettre en évidence les variations relatives de chaque paramètre au cours du temps.

Nous pouvons, de l'ensemble des données, dégager les faits suivants:

a) Poids des animaux (fig. 2-I).

Au cours des 7 semaines de carence partielle en chlore, le poids corporel des pondeuses n'évolue pas différemment de celui des témoins. I'écart entre le poids moyen des poules de chaque lot, qui existait avant le traitement, reste constant au cours du temps.

b) Production d'œufs (fig. 2-II).

Un régime à faible teneur en $\mathrm{Cl}$ fait apparaitre une chute très nette de la production d'œufs, puisque celle-ci ne représente plus après 7 semaines que 44 p. Ioo de la valeur initiale chez les animaux carencés contre 75 p. Ioo chez le lot témoin (tabl. 2 ).

L'analyse de variance ne montre aucune différence entre lots déficients; en revanche, la variation dans le temps est très hautement significative $(P<0,0005)$. L'analyse statistique des données de la dernière semaine montre enfin qu'à ce stade, tous les lots carencés ont une ponte significativement inférieure à celle du lot témoin, situation inverse de celle rencontrée en début d'expérience.

c) Poids de l'auf (fig. 2-III).

Il apparaît chez les 7 lots déficients en chlore, comparés au lot témoin, une diminution relative du poids de l'œuf qui se traduit, à la $6^{\text {e }}$ semaine, par un déficit de 

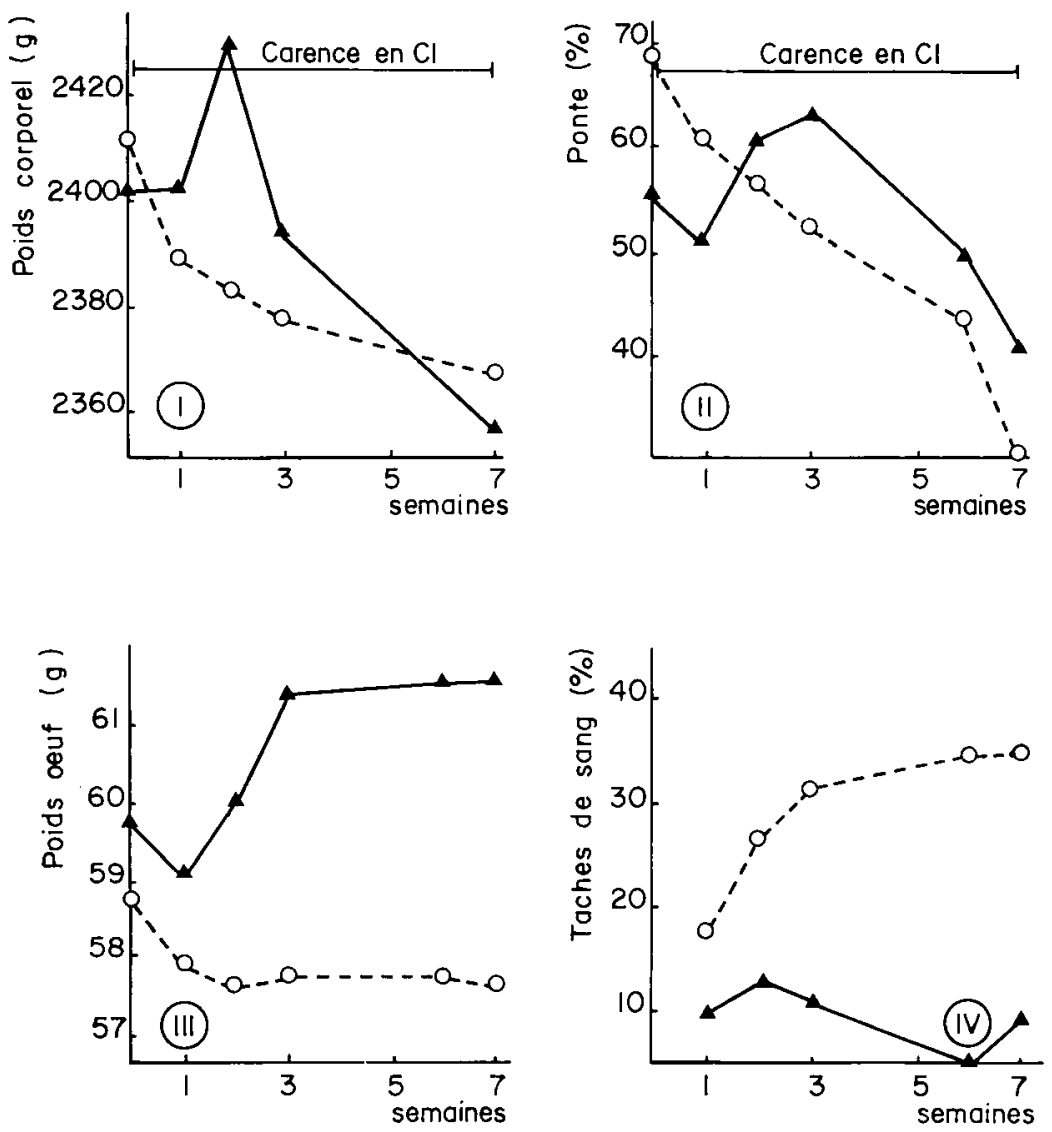

Fig. 2. - Déficience chlorée (o, I p. Ioo NaCl). Action sur le poids corporel des poules (I), l'intensité de ponte (II), le poids de l'ceuf (III) et la fréquence des taches de sang (IV)

$$
\begin{aligned}
& -\rightarrow \text { lot témoin }(0,24 \mathrm{p} \text {. Ioo } \mathrm{Cl}) \\
& \mathrm{o} \rightarrow-\rightarrow \text { lots déficients en } \mathrm{Cl}(0,06 \mathrm{p} \text {. Ioo } \mathrm{Cl})
\end{aligned}
$$

3.65 g (tabl. 2). En fait, l'effet essentiel du traitement semble être d'annuler l'augmentation du poids de l'œuf normalement liée au vieillissement de l'animal.

d) Fréquence des taches de sang (fig. 2-IV).

Les taches éventuellement présentes dans l'albumen de l'œuf (taches de " sang ou de "viande " que nous regroupons toutes ici sous la première appellation) sont un des facteurs importants de dépréciation de la qualité interne de l'œuf. La figure 2-IV donne la fréquence des œufs renfermant de tels défauts et il est surprenant de constater que la déficience chlorée entraîne une augmentation hautement significative de ce paramètre (tabl. 2). Après 6 semaines de traitement, cette fréquence est de $35 \mathrm{p}$. Ioo (contre $5 \mathrm{p}$. Ioo dans le lot témoin) soit 7 fois plus élevée; globalement l'accroissement de cette fréquence est de $225 \mathrm{p}$. Ioo sous l'action de la déficience en chlore. En outre l'analyse de variance montre que la réponse des poules à l'intérieur des lots est également différente $(\mathrm{P}<0,005)$. 

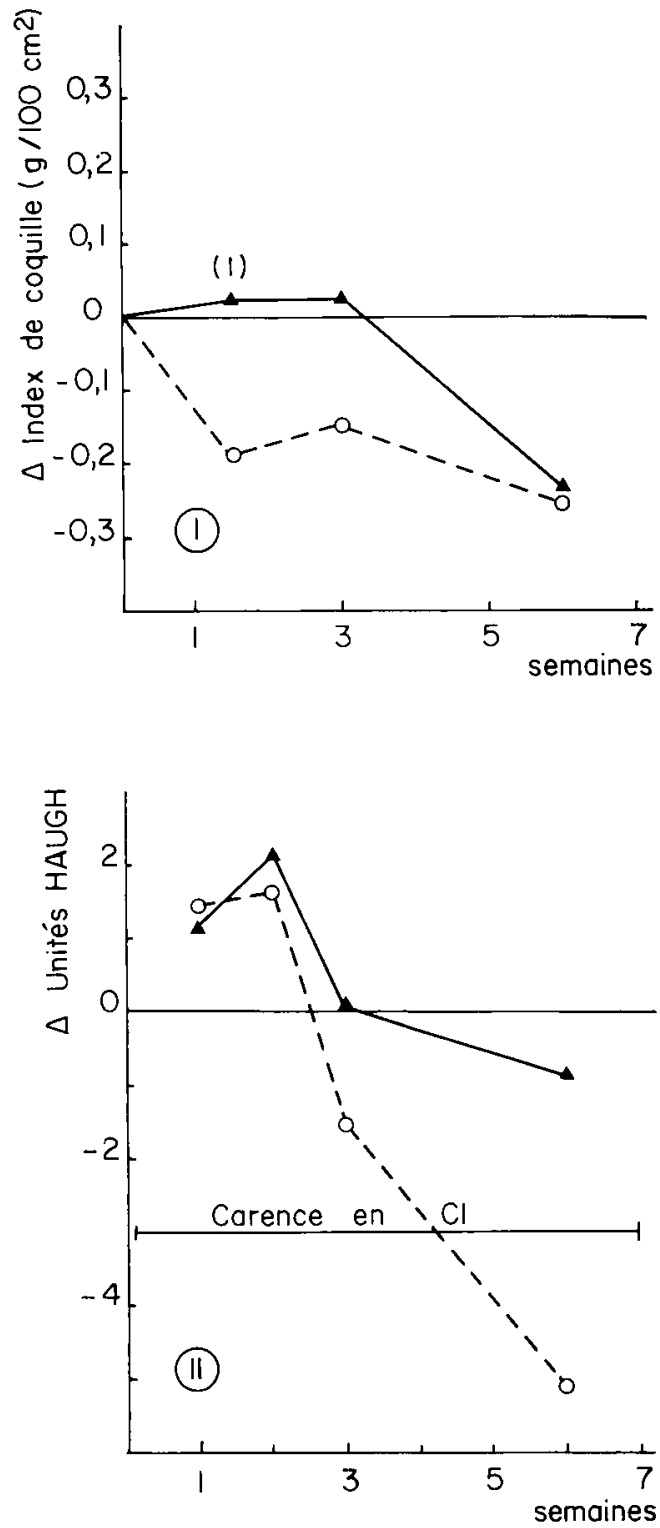

lis. 3. - Variations par rapport à la période pré-expérimentale de l'index de coquille (I) et de la qualité de l'albumen de l'ceuf (II) sous l'effet d'une déficionce chlorée (o, I p. Ioo NaCl) $(\mathrm{I})=$ moyenne des 2 premières semaines

$\triangle-\ldots$ lot témoin $(0,24$ p. Ioo Cl)

o - - o lots déficients en $\mathrm{Cl}(0,06 \mathrm{p}$. $100 \mathrm{Cl})$

e) Solidité des coquilles (fig. 3-I).

Comme tous les paramètres suivants relatifs à l'œuf, ce critère est étudié sous forme de variation par rapport à la période de pré-traitement. Sur cette base, l'analyse de variance démontre l'existence de différences significatives entre lots $(\mathrm{P}<0,005)$ qui peuvent être décomposées comme suit. 
ANIONS ALIMENTAIRES CHEZ LA POULE

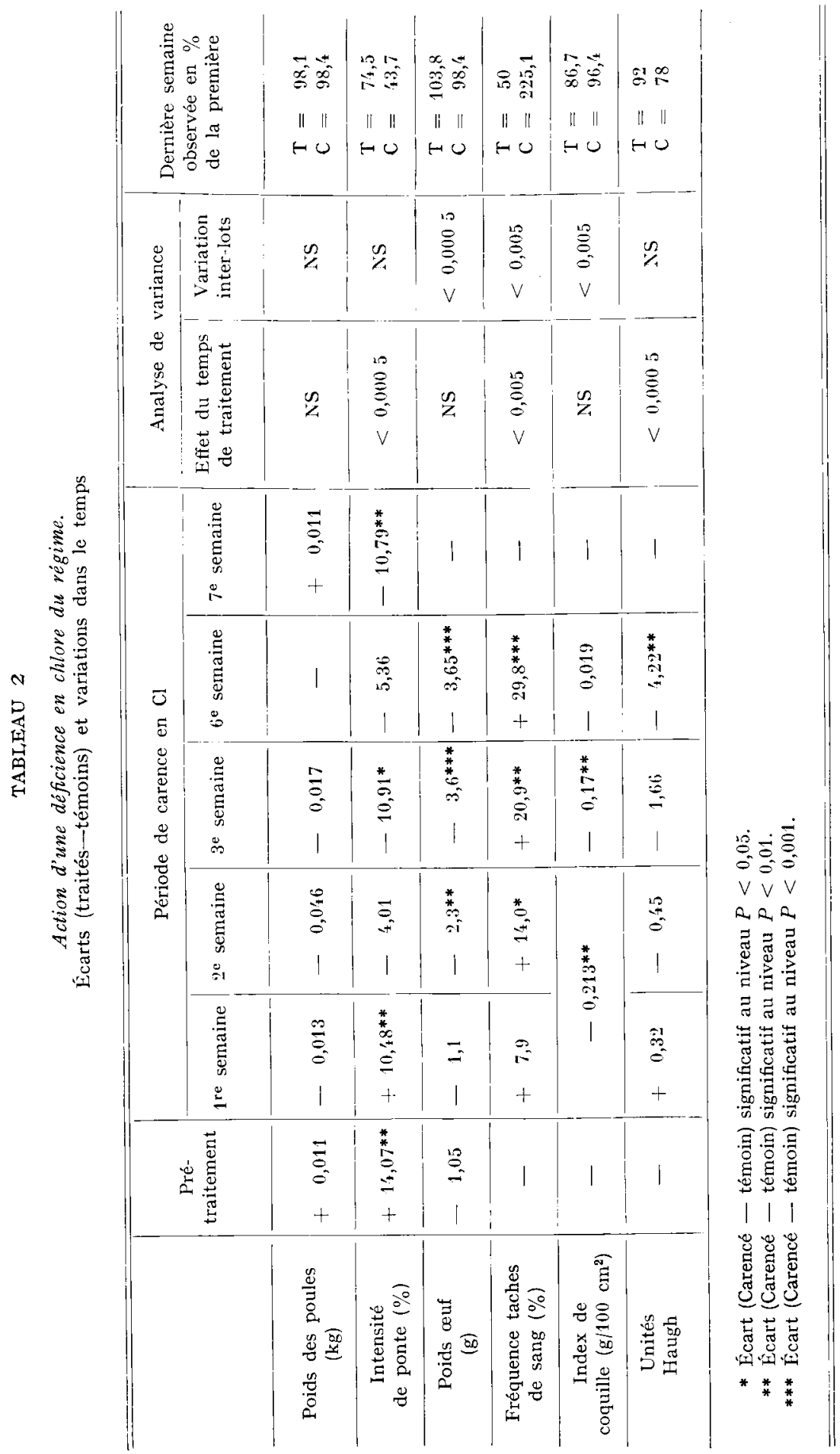


La comparaison des moyennes des deux premières semaines de traitement montre une diminution de solidité de coquille des lots traités par rapport au témoin égale à 0,2 I3 unité d'index. Par contre, entre la $I^{\text {re }}$ et la $6^{\mathbf{e}}$ semaine, la décroissance de l'index est proportionnellement moins importante pour les lots expérimentaux (- 0,068 soit 3,06 p. I00) que pour le témoin (-0,26 soit I3,3 p. I00) $(P<0,002$, tab1. 2).

Il semble donc que la déficience chlorée atténue la diminution normale d'index de coquille liée à l'âge de la pondeuse (effet symétrique de celui observé sur le poids de 1'œuf).

f) Qualité de l'albumen (fig. 3-II).

La restriction chlorée agit de façon identique sur tous les lots traités et entraîne une diminution de la qualité de l'albumen hautement significative dans le temps $(P<0,0005)$. Globalement, entre la $I^{\text {re }}$ et la $6^{\mathrm{e}}$ semaine, le score d'unités Haugh chute de 22 p. Ioo chez les animaux restreints contre 8 p. Ioo chez les témoins (tabl. 2). Cet effet défavorable du régime hypochloré est surtout visible à partir de la $3^{\mathrm{e}}$ semaine et apparaît donc opposé à celui exercé sur la solidité de la coquille.

\section{Expérience III. Supplémentation en chlorure et sulfate de sodium}

Nous avons reporté au tableau 3 les résultats relatifs à cette période sous forme d'écarts entre la fin de supplémentation et le début du traitement (fin de déficience chlorée). Les seuils de signification obtenus en comparant les moyennes des lots I et 5,2 et 6,3 et 7 et 4 et 8 figurent également sur ce tableau (lots identiques 2 à 2 pour la teneur en chlore mais contenant des doses différentes de $\mathrm{Na}_{2} \mathrm{SO}_{4}$ ).

\section{a) Poids des animaux.}

L'apport de différents mélanges de chlorure et de sulfate de sodium affecte de façon significative le gain de poids vif des pondeuses. Ainsi, pour une faible teneur en chlore du régime $(0, \mathrm{I}$. Ioo de $\mathrm{NaCl}$, lots I et 5$)$, le gain de poids diminue lorsque la teneur en sulfate augmente mais avec une forte teneur en chlore $(0,4 \mathrm{p}$. Ioo de $\mathrm{NaCl}$, lots 4 et 8$)$, une dose élevée de sulfate améliore le gain de poids $(P<0,02)$.

\section{b) Production d'cufs.}

Les valeurs de ce paramètre reportées au tableau 3 montrent clairement que la production d'œufs reprend sous l'effet des différentes supplémentations en sel de sodium, à tel point qu'en fin d'expérience tous les lots traités ont des intensités de ponte supérieures à celle du lot témoin $\left(n^{\circ} 4\right)$, situation inverse de celle rencontrée en fin de déficience chlorée (Expérience II).

Pour les lots I et 5 la production augmente alors que la teneur de l'aliment en chlore n'a pas changé (o,I p. roo de $\mathrm{NaCl})$; l'apport de sulfate suffit pour faire reprendre la ponte. Par contre, le supplément de sulfate du lot 5 par rapport au lot I n'a aucun effet. La constatation est la même entre les lots 2 et 6 ou 3 et 7 . Entre les lots 4 et 8 , la différence est très nette, mais il serait risqué d'y attacher trop d'importance, car il semble que malgré la répartition au hasard des animaux, le lot témoin soit nettement inférieur aux autres.

Nous pouvons tirer trois conclusions de ces résultats. D'une part, un apport de 
ANIONS ALIMENTAIRES CHEZ I,A POULE

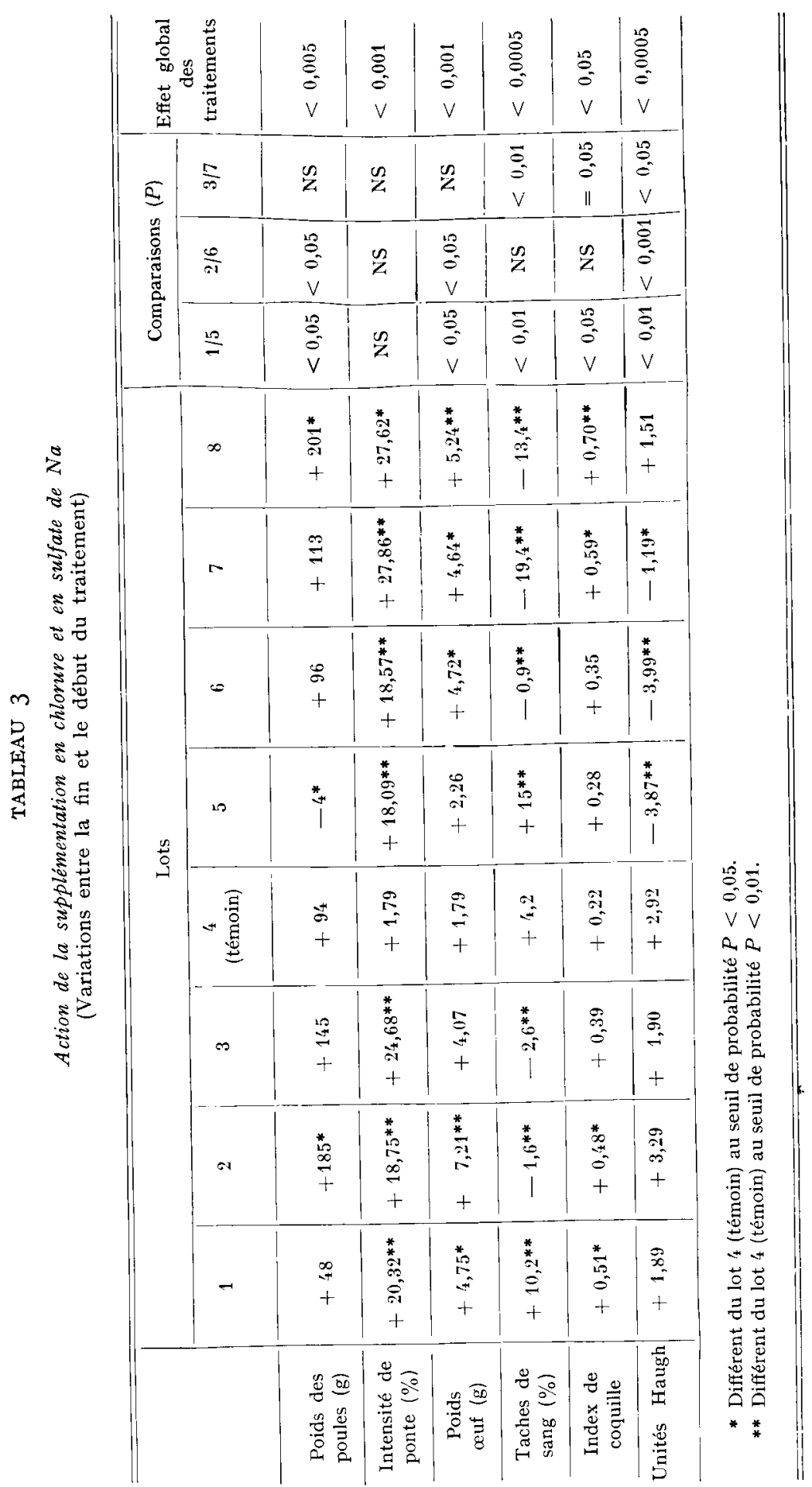


sulfate restaure partiellement la production malgré le maintien de la déficience chlorée. D'autre part, pour des régimes isochlorés, un apport supplémentaire d'ions $\mathrm{SO}_{4}{ }^{=}$ne modifie pas la ponte. Enfin, il semble qu'un minimum de chlore équivalent à $0,2 \mathrm{p}$. Ioo de $\mathrm{NaCl}$ soit nécessaire pour maintenir une bonne production.

c) Poids de l'ceuf.

Tous les lots (sauf 3 et 5) présentent un gain de poids d'œuf significativement supérieur à celui du lot 4 (témoin). Le gain maximum est obtenu avec $0,2 \mathrm{p}$. Ioo de $\mathrm{NaCl}$ et 0,24 p. Ioo de $\mathrm{Na}_{2} \mathrm{SO}_{4}$ (lot 2). Nous constatons que, comme l'intensité de ponte, le poids de l'œuf augmente chez les animaux qui n'ont reçu qu'un supplément de sulfate sans réajustement de la teneur du régime en chlore (lot I).

La comparaison des effets obtenus avec différents apports de sulfate montre que, pour des teneurs en $\mathrm{NaCl}$ inférieures à $0,2 \mathrm{p}$. Ioo, une forte dose de sulfate est moins efficace qu'une faible dose (lots I et 5 ou 2 et 6 ). Par contre, avec 0,4 p. Ioo de $\mathrm{NaCl}$, une dose de 0,48 p. Ioo de sulfate améliore le poids de l'œuf ; avec 0,3 p. Ioo de $\mathrm{NaCl}$, la quantité de sulfate est indifférente.

d) Taches de sang.

Tous les lots recevant du sulfate de sodium présentent en fin de traitement une fréquence de taches de sang supérieure à celle du lot témoin $(P<0,00 I)$ comme l'indiquent les valeurs suivantes:

\begin{tabular}{|c|c|c|c|c|c|c|c|c|}
\hline Lots & 1 & 2 & 3 & $\stackrel{4}{4}$ & 5 & 6 & 7 & 8 \\
\hline Taches de sang (\%) & $41, \mathbf{k}$ & 31,7 & 23,5 & 13,5 & $5: 5$ & 31,1 & 26,0 & 21,6 \\
\hline
\end{tabular}

Si nous ne considérons que la variation de fréquence entre la fin de la déficience chlorée et celle de la supplémentation anionique (tab1. 3) nous constatons que, pour une teneur faible en chlorure, soit o, I p. Ioo de $\mathrm{NaCl}$, la fréquence des taches augmente avec la teneur en sulfate du régime (lots I et 5). Par contre, lorsque la teneur en chlorure est plus élevée cette fréquence diminue et ce, d'autant plus que la quantité de sulfate ajoutée est importante (comparer les lots 3 et 7 et les lots 4 et 8). Un régime pauvre en chlorure favorise donc, comme dans l'expérience II, 1'apparition des taches de sang, effet potentialisé par les sulfates; avec un régime normalement pourvu en chlorure l'effet des sulfates est au contraire bénéfique.

e) Index de coquille.

Comparés au lot témoin (4), les lots 3, 5 et 6 ne montrent pas d'amélioration significative de la solidité de la coquille. Avec une faible teneur du régime en chlorure (lots I et 5), une faible dose de sulfate a un effet positif $(P<0,05)$. Pour les lots 2 et 6 la tendance est la même, mais non significative. Avec des apports de chlorure élevés : 0,3 p. Ioo (lots 3 et 7) et 0,4 p. Ioo (lots 4 et 8), l'effet est inversé et une forte teneur en sulfate entraîne un accroissement de solidité des coquilles. Nous retrouvons donc, comme précédemment, une interaction entre les niveaux alimentaires des deux anions étudiés. 
f) Qualité de l'albumen.

Nous observons, en fin de supplémentation anionique, une importante diminution de la qualité de l'albumen des lots 5, 6 et 7 recevant de fortes doses de sulfate de sodium. Pour une faible teneur de l'aliment en chlorure (lot I et 5) une forte teneur en sulfate accentue l'action défavorable de la déficience chlorée, alors qu'une faible teneur en diminue l'effet $(P<0$,or).

A des niveaux supérieurs de $\mathrm{NaCl}$ (o,2 et 0,3 p. Ioo), l'effet des ions sulfate va dans le même sens $(P<0,002)$.

\section{DISCUSSION}

Les travaux de Burns et al. (I952) et MAC DoNALD (I962) ont bien mis en évidence le rôle global du chlorure de sodium sur la production de poules pondeuses; toutefois ces auteurs se sont surtout attachés à démontrer l'effet propre du sodium, considérant que le chlore n'était pas un facteur limitant. Les résultats que nous rapportons ici démontrent que dans un régime normosodé, une restriction de l'apport en chlore peut affecter de façon nette les caractéristiques de l'œuf et la production des animaux. Il en est de même d'un apport de sulfate de sodium.

La première expérience démontre que la suppression totale du chlore alimentaire chez les pondeuses entraîne, en trois semaines environ, un arrêt quasi total de la production en même temps qu'un amaigrissement progressif des animaux (fig. I-II et I-I). Il n'en est plus tout à fait de même lorsque nous apportons $0, I$ p. Ioo de $\mathrm{NaCl}$ (fig. $2-\mathrm{II}$ et $2-\mathrm{I}$ ) ; à ce niveau, l'amaigrissement disparaît mais, après sept semaines de traitement, la production d'œufs reste inférieure à celle du lot témoin. Les résultats

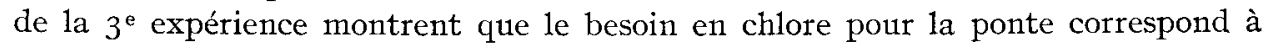
0,2 p. Ioo de $\mathrm{NaCl}$ et que, en deçà de cette valeur, un apport de 0,36 p. Ioo de $\mathrm{Na}_{2} \mathrm{SO}_{4}$ permet d'atténuer les effets de la déficience chlorée. Ce résultat confirme celui obtenu par Burns et al. (1952) qui, selon les mêmes critères, fixait à o, I9 p. Ioo le besoin en $\mathrm{NaCl}$ des poules pondeuses.

L'action dépressive de la restriction chlorée sur le poids de l'œuf (fig. 2-III) confirme quant à elle les observations de MAC DoNALD (I962) relatives à une carence globale en $\mathrm{NaCl}$ et montre de plus l'action propre du chlore. Le gain de poids d'œuf maximum est obtenu, après déficience chlorée, avec $0,2 \mathrm{p}$. Ioo de $\mathrm{NaCl}$ et $0,24 \mathrm{p}$. Ioo de $\mathrm{Na}_{2} \mathrm{SO}_{4}$. De plus, l'étude de ce critère met en évidence une interaction entre chlorures et sulfates du régime, les seconds n'étant intéressants à dose élevée que si les premiers sont fournis en quantité suffisante.

L'étude de ces mêmes traitements sur l'équilibre acido-basique du sang montre que la restriction de l'apport alimentaire de chlore entraîne l'apparition d'une alcalose métabolique pratiquement compensée mais aggravée par l'apport de sulfates (MoNGir, SAuveur et AMin, I97I). Une teneur en chlore du régime équivalente à 0,2 p. IOO de $\mathrm{NaCl}$ suffit à rétablir un équilibre acido-basique et un ionogramme sanguin normaux ; ces critères aboutissent par conséquent à une définition du besoin en chlore identique à celle obtenue par des paramètres zootechniques.

On sait que la formation de la coquille est liée à l'équilibre acido-basique de la poule (voir MONGIN, Ig68 a) et que par ailleurs celui-ci affecte la qualité de l'albumen mesurée en unités Haugh (SAUvEUR, I97o). 
Sans vouloir rappeler une nouvelle fois tous les détails de ces phénomènes, disons qu'en règle générale une acidose métabolique est défavorable à la solidité de la coquille mais bénéfique pour l'albumen. Les effets exercés par une alcalose respiratoire due à une hyperventilation d'origine thermique sont les mêmes (MUELLER, I966). A l'opposé, une hypercapnie chronique se révèle favorable à la coquille (MonGIN, I968 b) mais néfaste pour la qualité du blanc (SAUVEUR, I968).

Il semble donc, en résumé, que, quel que soit le sens de variation đu $\mathrm{pH}$ sanguin, la solidité de la coquille augmente lorsque la teneur du sang en bicarbonate disponible est élevée; la qualité de l'albumen diminue dans les mêmes conditions. Les résultats de 1'expérience II sont en accord avec cette hypothèse. Nous avons v1 en effet que la déficience chlorée agit de façon diphasique sur la solidité de la coquille (fig. 3-I). En début de carence l'index de coquille diminue mais, après six semaines de traitement, ce dernier se révèle finalement bénéfique, en compensant l'effet défavorable du vieillissement de la poule.

Il convient par conséquent de distinguer dans l'alcalose hypochlorémique la phase aiguë de la phase chronique comme dans le cas d'une hypercapnie (MoNGIN et SAUVEUR, I970; SAUVEUR et MONGIN, à paraître). L'animal soumis à une déficience chlorée soudaine développe une alcalose due à une régénération accrue de bicarbonate par le rein (RICHET et LISSAC, I963) ; les charges électronégatives ainsi créées compensent le déficit en chlore au niveau sanguin. En phase chronique, cet équilibre ionique est stabilisé puisque nous observons un accroissement de bicarbémie de 9,8 méq/1 pour un abaissement de chlorémie de io,6 méq/1 (Mongin, Sauveur et Amrn, I971). Les ions bicarbonate en excès produits au niveau rénal peuvent alors tamponner les ions $\mathrm{H}+$ libérés lors de la formation de la coquille (HODGES et LörCHER, I967).

Il reste également possible que l'amélioration relative de la solidité de la coquille en fin de déficience chlorée soit liée en partie à la stabilisation du poids de l'œuf observée chez les lots déficients en chlore.

L'effet des ions sulfatesur la solidité des coquilles est plus complexe. Dans legroupe I (recevant peu ou pas de sulfate), l'index de coquille augmente avec la teneur en sulfate du régime, alors que dans le groupe 2 , il augmente lorsque la teneur en sulfate diminue. Si nous rappelons également que, dans chaque lot, les taux de sulfate et de chlorure varient en sens inverse, nous pouvons en déduire que le rapport entre les deux anions doit intervenir. Le tableau 4 montre en particulier que la solidité de la coquille croît comme la teneur en sulfate jusqu'à un taux de 0,48 p. I00, mais qu'ensuite elle diminue si la teneur en sulfate augmente.

\section{TABLEAU 4}

Comparaison entre l'augmentation de la solidité de la coquille et celle de la teneur en sulfate de sodium de l'aliment

\begin{tabular}{|c|c|c|c|c|c|c|c|c|}
\hline Teneur en sulfate de & 0 & 0121 & 0.242 & 0363 & 0.484 & 0,605 & 0.726 & 0847 \\
\hline Lots ............. & 4 & 3 & 2 & 1 & 8 & 7 & 6 & 5 \\
\hline $\begin{array}{l}\text { Augmentation de la } \\
\text { solidité de coquille. }\end{array}$ & $+0,22$ & $+0,39$ & $+0,48$ & $+0,51$ & $+0,70$ & $+0,59$ & $+0,35$ & $+0,28$ \\
\hline
\end{tabular}


L'action des divers traitements sur la qualité de l'albumen mesurée en unités Haugh est également conforme aux données déjà acquises sur les rapports entre équilibre acido-basique et qualité du blanc. En effet nous observons dans la phase chronique de la déficience chlorée (Exp. II) une diminution hautement significative de cette qualité et, dans l'Expérience III, l'apport de grandes quantités de sulfate (qui aggravent l'alcalose hypochlorémique) s'accompagne d'une baisse supplémentaire des unités Haugh (lots 5 et 6 ). Pratiquement un rapport chloré équivalent à 0,2 p. Ioo de $\mathrm{NaCl}$ paraît indispensable au maintien de la qualité de l'albumen.

D'autre part, l'analyse de la composition électrolytique du blanc, effectuée simultanément aux mesures d'unités Haugh (SAUveur, Mongin et Amin, à paraître) confirme l'existence d'une relation positive entre qualité du blanc et teneurs en $\mathrm{Cl}$ et $\mathrm{K}+$ et d'une relation inverse entre cette même qualité et la teneur de l'albumen en $\mathrm{Na}^{+}$. Ce résultat est en accord avec ceux obtenus lors d'acidoses métaboliques (SAUVEUR, I970).

\section{Problème particulier des taches de sang}

Les taches présentes dans l'albumen de l'œuf ou adhérentes au jaune sont souvent constituées de caillots sanguins dus à une hémorragie folliculaire antérieure à l'ovulation (NALBANDOV et CARD, I944). Elles pourraient également provenir de l'isthme et de l'utérus ; dans ce cas, elles sont riches en calcium et fluorescentes en lumière ultraviolette.

Sans pouvoir préciser de quel type il s'agit, nous avons observé dans l'Expérience II qu'une déficience chlorée accroit considérablement leur fréquence; les résultats obtenus dans la dernière partie de cette étude montrent de plus que la fréquence élevée de ces taches, liée au déficit en chlore, peut être accrue par 1'apport de sulfate (lots I et 5). Au contraire, lorsque l'apport de chlore est élevé (lots 4 et 8 ) les ions sulfate semblent exercer un effet favcrable.

L'interprétation de ces résultats s'avère difficile. NALBANDOV et CARD montrent qu'une élévation du $\mathrm{pH}$ de l'albumen accélère la décoloration des taches dites " de sang "; il est probable que, dans nos conditions expérimentales, un tel accroissement de $\mathrm{pH}$ est intervenu mais ceci n'explique pas l'augmentation de la fréquence. LAHELLEC (I965) fait d'autre part remarquer que les taches sont systématiquement plus fréquentes dans les œufs à coquille colorée, tels que ceux étudiés ici, mais l'auteur ne dit rien d'un rôle possible du chlore.

Nous en sommes, par conséquent, réduits à supposer que l'hypochlorémie a pu agir soit sur la fragilité des capillaires ovariens, soit au niveau des tissus de l'isthme suivant un mécanisme qui, dans les deux cas, reste à déterminer.

\section{CONCLUSION}

D'un point de vue pratique, les résultats obtenus ici en distribuant à des poules pondeuses des quantités variables de chlorure et de sulfate de sodium, peuvent se résumer ainsi :

- le besoin en chlore de la poule pondeuse apparaît voisin de o,2 p. Ioo de $\mathrm{NaCl}$; avec des taux alimentaires inférieurs, la déficience chlorée se traduit par une baisse 
de la production, du poids et de la qualité interne de l'œuf, essentiellement par une apparition d'une quantité importante de taches de sang;

- des teneurs en sodium alimentaire aussi élevées que 0,3I p. roo n'ont aucun caractère de nocivité. L,es effets dépressifs d'un excès de chlorure de sodium observés par LöRCHER et al. (I964) sont le fait du chlore plus que celui du sodium.

- un excès ce chlore est défavorable à la solidité de la coquille ;

- il existe une interaction entre les ions $\mathrm{Cl}^{-}$et $\mathrm{SO}_{4}{ }^{-}$du régime et les seconds ne doivent être éventuellement apportés que si les premiers sont présents en quantité suffisante ;

- avec un niveau alimentaire de $\mathrm{NaCl}$ voisin de $0,2 \mathrm{p}$. IoO, une supplémentation en $\mathrm{Na}_{2} \mathrm{SO}_{4}$ de $0,25 \mathrm{p}$. Ioo permet d'améliorer la qualité de la coquille sans nuire à celle de l'albumen.

Reçu pour publication en décembre $19 \% 0$.

\title{
SUMMARY
}

\author{
EFFECT OF DIFFERENT LEVELS OF SODIUM CHLORIDE \\ AND SODIUM SULFATE IN THE DIET \\ OF LAYING HENS UPON EGG PRODUCTION AND EGG QUALITY
}

The purpose of this study was to measure the effect of different levels of chloride and sulfate in the diet on egg production and egg quality when the sodium content was held constant. These trials were conducted with Rhode Island Red $\times$ Wyandotte hens; we measured body weight, egg production, egg weight, shell strength and albumen quality.

With a chloride free synthetic diet containing a normal quantity of sodium (as $\mathrm{NaHCO}_{3}$ ), the loss of body weight averaged 4 oo $g$ after five weeks and egg production had practically ceased after four weeks (fig. I).

With a diet containing $\mathrm{CaCl}_{2}$ to provide a level of chloride equivalent to o. I p. Ioo of $\mathrm{NaCl}$ (fig. 2 and 3), we observed a decrease in egg production of $10.8 \mathrm{p}$. Ioo and in egg weight of $3.7 \mathrm{~g}$ relative to the control diet. The albumen quality was also reduced for 4.2 Haugh units. (The control diet contained $\mathrm{CaCl}_{2}$ providing chloride ion equivalent to o,4 p. roo $\mathrm{NaCl}$ ). The chloride deficient diet seemed to enhance shell strength after two weeks while the frequency of blood spots increased sevenfold after six weeks.

After the chloride deficient period, different levels of sodium chloride and sodium sulfate were added to the diet (tabl. I $d$ ). It appeared that the minimum chloride requirement of the laying hen was provided by about $0.2 \mathrm{p}$. I oo of $\mathrm{NaCl}$ and that these two anions interact. When the chloride content was equivalent to 0.2 p. 100 of $\mathrm{NaCl}$, a supplementation by 0.25 p. Ioo of $\mathrm{Na}_{2} \mathrm{SO}_{4}$ increased shell strenght without any effect upon the other parameters. On the contrary in a chloride deficient diet (containing less than $0.2 \mathrm{p}$. Ioo of $\mathrm{NaCl}$ ) the addition of sulfate accentuated the adverse effects of chloride deficiency on internal egg quality (Haugh units and blood spots).

As chloride deficiency also causes a metabolic alkalosis (Mongin, Sauveur and Amin, I97r) these results are discussed in relation to the effect of acid-base balance upon eggshell and albumen formation.

\section{RÉFÉRENCES BIBLIOGRAPHIQUES}

Burns C. H., Cravens W. W., Phillips P. H., I952. The requirement of breeding hens for sodium chloride. Poultry Sci., 31, 302-306.

Mac Donald H. W., I962. Salt deficiency in laying diets. Proc. 12th World's Poult Congr., $212-218$.

HaUgh R. E., I937. The Haugh unit for measuring egg quality. U. S. Egg Poult, Mag., 43, 552-555.

Hodges R. D., Lörcher K., I967. Possible sources of the carbonate fraction of egg shell calcium carbonate. Nature, 216, 609-6ro. 
LaHellec Cécile, I965. Contribution à l'étude des variations qualitatives de l'œuf de poule au cours d'une saison de ponte. Bull. Inform. Station expér. Avicult. Ploufragan, 5 (30).

Lörcher K., Diamanstein T., Kobow J., r964. Der Einfluss von Chloridionen auf die Eischalenstabilität. Z. Tierphysiol. Tiernähr. Futtermittelkde, 19, 228-236.

Mongin P., I965. Index de solidité de coquille de l'œuf. Ses significations. Sa précision. Ann. Zootech., 14, 3I9-325.

Mongin P., I $968 a$. Role of acid-base balance in the physiology of egg shell formation. World's Ponit. Sci. J., 24, 200-230.

Mongin P., I 968 b. Keeping laying hens in $\mathrm{CO}_{2}$ enriched atmosphere. The action of $\mathrm{CO}_{2}$ on the shell quality. 3rd European Poult. Conf. Jerusalem. Work Group no 7, p. 5 (abstr.).

Mongin P., Savveur B., I97o. Équilibre acido-basique du sang et composition ionique du plasma chez la poule en hypercapnie chronique. Ann. Biol. anim. Bioch. Biophys., 10, no hors-série 2, I 4 I-I 50.

Mongin P., Sauveur B., Amin M., ig7i. Alcalose hypochlorémique chez la poule pondeuse. Ann. Biol. anim. Bioch. Biophys. (sous presse).

Mueller W. J., I966. Effect of rapid temperature changes on acid-base balance in shell quality (abstr.). Poultry Sci., 45, I109.

Nalbandov A. V., Card L. E., I944. The problem of blood clots and meat spots in chicken eggs. Poultry Sci., 23, $270-180$.

Richet G., Lissac J., 1963. L'acidurie de l'alcalose métabolique n'est qu'un faux paradoxe. Rev. franc. Etudes clin. et biol., 8, $753-756$.

SAUveur B., I968. Keeping laying hens in $\mathrm{CO}_{2}$ enriched atmosphere. The action of $\mathrm{CO}_{2}$ on the albumen quality. 3rd European Poult. Conf. Jerusalem. Work Group no 7 , p. 5 (abstr.).

SaUveur B., I970. Acidoses métaboliques expérimentales chez la poule pondeuse. II. Action sur la composition minérale de l'albumen de l'ouf. Ann. Biol. anim. Bisch. Biophys., 10, 8I-Ioo. 